\title{
LA JURISPRUDENCIA DEL TRIBUNAL SUPREMO ESPAÑOL ES CLARA: SOLICITAR LA RENOVACIÓN DEL PASAPORTE ESPAÑOL ANTE EL CONSULADO ES INDICATIVO DE LA VOLUNTAD DE CONSERVACIÓN DE LA NACIONALIDAD ESPAÑOLA (NOTAS A LA SENTENCIA DEL TRIBUNAL SUPREMO DE 1 DE JUNIO DE 2020)
}

\author{
THE JURISPRUDENCE OF THE SPANISH SUPREME COURT \\ IS CLEAR: REQUESTING THE RENEWAL OF THE SPANISH \\ PASSPORT AT THE SPANISH CONSULATE IS INDICATIVE \\ OF THE WILL TO CONSERVATE THE SPANISH NATIONALITY \\ (NOTES TO THE JUDGMENT OF THE SUPREME COURT \\ OF JUNE 1, 2020)
}

\author{
LeRdys S. Heredia SÁnchez \\ Profesora Ayudante de Derecho internacional privado \\ Universidad Miguel Hernández de Elche \\ ORCID: 0000-0003-1092-8868
}

Recibido: 25.11.2020 / Aceptado: 14.12.2020

DOI: https://doi.org/10.20318/cdt.2021.6001

\begin{abstract}
Resumen: El presente comentario se refiere a la Sentencia del Tribunal Supremo español de fecha 1 de junio de 2020, por la que se resuelve un recurso de casación que trae como base la aplicación del artículo 24.3 del Código Civil, en cuanto a la causa de pérdida de la nacionalidad española de los nacidos en el extranjero de progenitor español también nacido fuera de España, por falta de declaración de conservación. Esta sentencia va en la misma línea que su antecesora, la dictada por el alto tribunal, el 19 de diciembre de 2019, y que ha venido a allanar el camino para evitar la pérdida de la nacionalidad a los españoles nacidos en el extranjero, al entender que con la mera solicitud de la renovación del pasaporte, se cumple con lo dispuesto en nuestro Código Civil.

Palabras clave: nacionalidad española, declaración de conservación, renovación de pasaporte, solicitud de conservación, pérdida de la nacionalidad.

Abstract: This paper analyzes the Spanish Supreme Court Judgment of June 1, 2020, which resolves an appeal in cassation based on the application of Article 24.3 of the Civil Code regarding the cause of loss of Spanish nationality for those born abroad to a Spanish parent who was also born outside of Spain, due to the lack of a declaration of retention of Spanish nationality. This ruling is in line with its predecessor, the one issued by the Supreme Court on December 19, 2019, which has opened the way for avoiding the loss of nationality to Spaniards born abroad, since it is understood that by merely applying for a passport renewal, the provisions of our Civil Code are complied with.
\end{abstract}

Keywords: lost of nationality, Spanish nationality, passport renewal, Spanish Supreme Court Judgment. 
Sumario: I. Introducción. II. Los antecedentes de hecho y la decisión que da lugar al recurso de casación. III. Algunas consideraciones sobre la causa de pérdida de la nacionalidad española por ausencia de declaración de conservación de la nacionalidad, cuando se trata de españoles nacidos en el extranjero de progenitores nacidos fuera de España. IV. Valoración global de la doctrina del Tribunal Supremo español para evitar la pérdida de la nacionalidad española de los españoles de origen nacidos en el extranjero, de acuerdo con el artículo 24.3 del Código Civil. V. Comentario final.

\section{Introducción}

1. Es objeto del presente comentario la Sentencia del Tribunal Supremo, de 1 de junio de $2020^{1}$ (en adelante, STS) que da continuidad a la interpretación flexible del articulo 24.3 del Código Civil (en adelante, CC) introducida por la STS 696/2019, de 19 de diciembre de $2019^{2}$ y que ha venido a dejar sentadas las bases para evitar la pérdida de la nacionalidad española de origen a los españoles nacidos en el extranjero, de progenitor español y que también ha nacido fuera de España.

2. El tema que aborda esta sentencia ha ganado un gran protagonismo jurídico en este último año, ya que el status quo sobre el que descansaba la doctrina administrativa desde el año 2002 (vinculando la declaración formal de conservar la nacionalidad española ante autoridad registral, para no perderla, por parte de quienes siendo españoles de origen y habiendo nacido en el extranjero venían obligados a ello) se ha visto removido en sus cimientos por la flexibilidad que ahora se acepta a partir del hecho social y habitual, que supone la solicitud de renovación del pasaporte español ante las autoridades consulares españolas en el extranjero; entendiendo que esta acción comporta de manera inequívoca la intención de la persona solicitante de querer ser española. Así, el TS invita a los operadores jurídicos a estudiar desde otra perspectiva, el Derecho español de la nacionalidad y a interpretar de manera flexible la exigencia recogida en el CC.

3. Para desarrollar estas notas se analizan, en primer lugar, los antecedentes de hecho y la decisión que da lugar al recurso de casación resuelto por la STS (II); a continuación, se realizan algunas consideraciones sobre la causa de pérdida de la nacionalidad española por ausencia de declaración de conservación de la nacionalidad, cuando se trata de españoles nacidos en el extranjero de progenitores nacidos fuera de España, la cual fue introducida en nuestro ordenamiento jurídico en el año 2002 (III); para, posteriormente, realizar algunas consideraciones acerca de la renovada interpretación del artículo 24.3 CC en torno a esta causa de pérdida de la nacionalidad española (IV); y por último, unas breves consideraciones finales, a modo de conclusión (V).

\section{Los antecedentes de hecho y la decisión que da lugar al recurso de casación}

4. Los hechos que dan lugar a la interposición del recurso de casación que resuelve la STS analizada son los siguientes: el recurrente, nacido en Bogotá (Colombia), hijo de madre española también nacida en Colombia, obtuvo en el año 2007 su pasaporte español, expedido por el Consulado General de España en Bogotá, con validez hasta el 22 de abril de 2012 y en el propio año 2012 el mismo Consulado renovó el pasaporte con fecha de expiración el 10 de abril de 2017.

Sin embargo, el 7 de septiembre de 2012, el Consulado General requirió al recurrente para que devolviera el pasaporte expedido en abril de ese mismo año (tras la segunda renovación) y el Encargado del Registro Civil Consular inició el expediente para declarar la pérdida de la nacionalidad española. Con fecha 18 de julio de 2013 acuerda la inscripción de la pérdida de la nacionalidad española en el acta de nacimiento del interesado, en virtud del artículo $24.3 \mathrm{CC}$, al considerar que el mismo había hecho uso exclusivo de la nacionalidad colombiana, sin formular declaración expresa de conservación de la nacionalidad española.

\footnotetext{
${ }^{1}$ STS 1539/2020 - ECLI: ES:TS:2020:1539.

${ }^{2}$ STS 4072/2019 - ECLI: ES:TS:2019:4072.
} 
5. El interesado presenta recurso contra dicha resolución ante la Dirección General de los Registros y del Notariado (en adelante, DGRN) -actualmente renombrada Dirección General de Seguridad Jurídica y Fe Pública- que desestima el recurso interpuesto y confirma la resolución impugnada, la cual es impugnada en sede judicial y da lugar a dos pronunciamientos totalmente contradictorios: el primero, la sentencia del Juzgado de Primera Instancia que estima la demanda (en adelante, SJPI) y revoca la resolución de la DGRN, reconociendo la nacionalidad española de origen del recurrente; mientras que por el otro lado, la Audiencia Provincial (en adelante, AP) -en contra del criterio de la Fiscalía- revoca la SJPI en apoyo de los argumentos del Abogado del Estado en la línea inflexible que alude a que para no perder la nacionalidad española, la declaración exigida por el artículo $24.3 \mathrm{CC}$ debe ser expresa y necesariamente ante el Encargado del Registro Civil y, por tanto, que no puede tenerse como tal declaración la solicitud de renovación de pasaporte. ${ }^{3}$

6. En síntesis, son éstos los datos fácticos y jurídicos que ponen sobre el tapete, una vez más, la actuación controvertida del Consulado Español en Colombia, con respecto a la exigencia "mecánica" del requisito previsto por el artículo $24.3 \mathrm{CC}$ a efectos de evitar la pérdida de la nacionalidad española de los españoles nacidos en el extranjero, descendientes de españoles también nacidos en el extranjero y avalada por la DGRN de forma reiterada en base a una errónea interpretación de lo dispuesto en la citada norma del CC, lo que conlleva a entender que se realice por los interesados "una expresa declaración de voluntad de conservar la nacionalidad española" ante el encargado del Registro Consular, y que en el caso de que no lo haga "la pérdida de la nacionalidad se produce siempre de pleno derecho". ${ }^{4}$

\section{Algunas consideraciones sobre la causa de pérdida de la nacionalidad española por ausencia de declaración de conservación de la nacionalidad, cuando se trata de españoles nacidos en el ex- tranjero de progenitores nacidos fuera de España}

7. La ley 36/2002 de modificación del CC en materia de nacionalidad ${ }^{5}$ introdujo en nuestro ordenamiento jurídico una nueva causa de pérdida de la nacionalidad española a través del citado apartado 3 del artículo 24 CC, de cuyo tenor literal se hace depender el mantenimiento de la condición de español de origen a la "declaración expresa de conservación" ante el encargado del Registro Civil en un plazo de 3 años, contados desde la mayoría de edad, o de la emancipación, a quienes habiendo nacido en el extranjero, de progenitores españoles también nacidos en el extranjero, quieran conservar la nacionalidad española.

La finalidad, que no es expresa, pero que se infiere de su literalidad, es de la evitar la perpetuación de estirpes indefinidas de descendientes de españoles en el extranjero, con la consecuencia de que continúen siendo españoles, aunque no mantengan ninguna vinculación con España, e incluso -en mu-

\footnotetext{
${ }^{3}$ Se da la circunstancia práctica de que en el caso del Consulado español en Colombia, la organización consular interna de España atribuye a una Sección diferente de la encargada del Registro Civil, la expedición de pasaportes. Cuestión que es puesta de manifiesto en el apartado 4 de los antecedentes de hecho de la STS y que el juzgador de la AP utiliza como argumento para entender que no se produce negligencia alguna en la actuación del personal consular, cuestión ésta en la que -como veremos más adelante- entra el TS en sus razonamientos a favor de la estimación del recurso.

${ }^{4}$ Son abundantes los ejemplos de la asentada doctrina administrativa que durante años ha ido generando la DGRN -desacertada en muchos casos- y con disímiles resoluciones que encuentran su fundamento en las más variadas consideraciones, destacando especialmente los relativos a españoles nacidos en Colombia y Brasil. Vid por todas, sendas RDGRN de 3 de marzo de 2017 (hermanos nacidos en Porto Alegre, Brasil, hijos de española también nacida en Brasil); RDGRN de 15 de 17 de 2017 (señor nacido también en Porto Alegre, Brasil); RDGRN de 12 de septiembre de 2013 (señor nacido en Uruguay); RDGRN de 12 de enero de 2008 (mujer argentina a quien corresponde contar el plazo a partir de los 21 años por ser esa la edad fijada en Derecho argentino para alcanzar la mayoría de edad); RDGRN de 17 de junio de 2019 (mujer colombiana que incluso durante parte de su niñez residió en España, aunque nació en Colombia al igual que sus padres); RDGRN de 30 de abril de 2015 (señor colombiano nacido allí, de padre español también nacido en Colombia); entre otras muchas.

${ }^{5}$ BOE núm. 242, de 9 de octubre de 2002.
} 
chos casos- sin saber que son españoles. En definitiva, esta modificación supuso el "rescate" del antiguo artículo $26 \mathrm{CC}$, en la redacción dada por la Ley de 15 de julio de $1954 .^{6}$

Con anterioridad a su promulgación se había criticado la ausencia de una norma que impidiera que los nacidos en países iberoamericanos en tales condiciones únicamente pudieran perder la nacionalidad española mediante su renuncia expresa, así como que sus hijos y sucesivos descendientes, mientras no sobreviniera la renuncia, serían igualmente españoles. ${ }^{7}$

8. Con la introducción en el año 2002 del artículo 24.3 CC se estableció un importante obstáculo -como apunta Vela ToRRES- para dichas situaciones, pues al exigir a los nietos de emigrados españoles, de la segunda generación nacida en el país de acogida, una declaración de voluntad dentro de un plazo concreto para no perder la nacionalidad, implica la pérdida ex lege si no se realiza esa declaración, sin embargo, y en contraposición, los hijos de los emigrantes nacidos fuera de España, quedan excluidos de esta institución. ${ }^{8}$

9. Siguiendo el análisis de este supuesto de pérdida de la nacionalidad española realizado por Carrascosa GonzÁlez, podemos fijar los siguientes requisitos para que dicha pérdida se produzca: el progenitor del individuo debe ser español, nacido en el extranjero; las leyes del país donde reside el individuo le han de atribuir su nacionalidad; el individuo ha de haber nacido en el extranjero y debe residir en el extranjero; y por último, la declaración ha de realizarse en el plazo de 3 años contados desde la emancipación o la mayoría de edad. Todos los cuales, se dan en el supuesto de autos que da lugar al pronunciamiento de casación. ${ }^{9}$

10. En torno a esta exigencia legal del artículo $24.3 \mathrm{CC}$ es necesario destacar dos cuestiones: la primera, en cuanto a quién debe ser la autoridad encargada de practicar la mencionada inscripción; y la segunda, a qué hace referencia la forma solemne en la que tal manifestación de conservación ha de realizarse.

En cuanto a la primera cuestión, no cabe duda de que esta competencia recae sobre el Encargado del Registro Civil en el que consta el nacimiento del interesado, quien debe calificar y decidir si se cumplen o no los requisitos para que se produzca la pérdida y, en consecuencia, proceder a la inscripción de la misma. ${ }^{10}$

${ }^{6}$ Ley de 15 de julio de 1954 por la que se reforma el Titulo Primero del Libro Primero del Código Civil, denominado "De los españoles y extranjeros", BOE núm. 197 de 16 de julio de 1954.

${ }^{7}$ Era ésta una de las críticas dirigidas por un autorizado sector doctrinal a la regulación de la pérdida de la nacionalidad contenida en el artículo 24 CC, en la redacción dada por la Ley 18/1990, de 17 de diciembre, pues, tratándose de países iberoamericanos o alguno de los que citaba el artículo $24 \mathrm{CC}$, el no emancipado nunca incurría en causa de pérdida y al llegar a la emancipación, al no regir la doctrina del asentimiento voluntario, sólo su renuncia expresa provocaba la pérdida, y los hijos y sucesivos descendientes, mientras no sobreviniera su renuncia, serían igualmente españoles. La doctrina es abundante en este tema, pero merece destacar, en particular, el interesante estudio que aborda los problemas prácticos en materia de nacionalidad y la actuación del Registro Civil en España realizado por A. ÁlvAREz Rodríguez, Nociones básicas del Registro Civil y problemas frecuentes en materia de nacionalidad, Ediciones GPS, Madrid, 2015, pp. 245-269; con anterioridad, la misma autora abordó el análisis de esta figura en su obra ID, Nacionalidad española. Normativa vigente e interpretación jurisprudencial, Aranzadi, Madrid-Navarra, 2008, pp. 119-120.

${ }^{8}$ Vid P. Vela Torres, "Sentencia del Pleno de la Sala Primera (Civil) del Tribunal Supremo n. ${ }^{\circ}$ 696/2019, de 19 de diciembre", Diario La Ley, No 9584, Sección Jurisprudencia, 2 de Marzo de 2020.

9 Vid J. Carrascosa GonzÁlez, Derecho español de la nacionalidad. Estudio práctico, Comares, Granada, 2011, p. 219. El autor hace referencia a otras dos cuestiones cuando analiza los requisitos exigidos: por un lado, al hecho de que el precepto no distingue el tipo de filiación -biológica o adoptiva- entre el individuo y el progenitor español; lo que no puede ser de otro modo ya que para el Derecho español los hijos son iguales ante la Ley, con independencia del vínculo filial que les una a sus padres; y por otro lado, el autor hace referencia al requisito de aplicación temporal de la normativa, que al no tener carácter retroactivo, se produciría a partir de la entrada en vigor, la cual tuvo lugar el 9 de enero de 2003.

10 Tal y como es analizado por A. Álvarez Rodríguez, Nociones básicas del Registro Civil y problemas frecuentes en ...op cit, p. 262; y también por C. Parra Rodríguez/M. Giménez Bachman, (Dirs.), Nacionalidad y extranjería, Huygens, Barcelona, 2016, pp. 71-72; E. Fernandez Maciá (Dir)/ AA.VV, Nacionalidad y extranjería, Tirant lo blanch, Valencia, 2018, pp.53-54; A. Ortega Giménez (Dir)/ L. Heredia SÁnchez (Coord.), Manual práctico orientativo de Derecho de la Nacionalidad, Aranzadi, Navarra, pp.113-114. 
Con respecto a la segunda, las formalidades exigidas a dicha declaración -y en lo que hoy se centra el TS con una visión mucho más flexible- se exige que la misma ha de ser formulada en el plazo de 3 años contados desde la emancipación, si la hubiera, o bien desde la mayoría de edad. Ello implica que la persona interesada ha de cumplimentar y firmar un impreso que, en la práctica, se pone a disposición de los nacionales españoles al efecto de dejar constancia expresa y por escrito de la voluntad de conservación de la nacionalidad española, de cuyo contenido se deriva la inscripción marginal realizada por el Encargado del Registro Civil y cuya consecuencia jurídica más inmediata es el reconocimiento de un supuesto de plurinacionalidad a favor de esta persona, una vez que acceda al Registro Civil, dotando de efectos constitutivos a dicha inscripción. ${ }^{11}$

Como ahora veremos, es esta cuestión -la de carácter formal- la que ha puesto de relieve la necesidad de adaptar los criterios interpretativos del artículo 24.3 a unas líneas más flexibles, prácticas y respetuosas con la ciudadanía española de origen.

11. Antes de entrar al siguiente epígrafe de estas notas, es de obligatorio trámite (aludiendo a los términos procesales) dejar constancia de la crítica a la normativa española que establece las causas de pérdida de la nacionalidad. No es fácil de entender el sentido de esta causa de pérdida de la nacionalidad española de origen -la del artículo 24.3 CC- porque genera discriminación entre españoles de origen.

Ello se explica cuando constatamos la disparidad que causa el propio artículo $24 \mathrm{CC}$ al distinguir entre hijos de españoles de origen, nacidos de padres nacidos en España cuyos hijos conservarán la nacionalidad española, a no ser que usen "exclusivamente" otra nacionalidad ya atribuida antes de la emancipación o de la mayoría de edad, en cuyo caso seguirán conservando la nacionalidad española como regla general.

Sin embargo, los hijos nacidos en el extranjero de padres nacidos también en el extranjero, perderán "en todo caso" la nacionalidad española si no formulan la declaración de conservación antes comentada.

12. El resultado es claro: ciudadanos de origen de primera y ciudadanos de origen de segunda porque así lo ha decidido el legislador, cuando quizás basta con englobar en un solo supuesto la conexión entre el español residente en el extranjero que ostente desde la minoría de edad otra nacionalidad, y quiera conservar la española, que así lo manifieste; con ello desaparecería la discriminación con la que hoy nos encontramos por el sólo hecho evitar la perpetuación de la estirpe española por el mundo. ${ }^{12}$

\section{Valoración global de la doctrina del tribunal supremo español para evitar la pérdida de la nacionalidad española de los españoles de origen nacidos en el extranjero}

13. Con respecto a la aplicación del artículo $24.3 \mathrm{CC}$, el TS se plantea nuevamente si la declaración de conservar la nacionalidad debe ser expresa y en concreto -en relación con el caso enjuiciado- si la solicitud de renovación del pasaporte dirigida al Consulado Español en Colombia tiene tal significado; cuestión que ya no es nueva para el juzgador, por lo que para resolver este asunto, trae a colación la STS 696/2029, de 19 de diciembre, que ha abierto la puerta al nuevo escenario que hoy tenemos. ${ }^{13}$

\footnotetext{
${ }^{11}$ Así lo entiende la doctrina española de forma generalizada considerando que esta inscripción tiene un carácter obligatorio. Vid por todos, M. Peña, "De los españoles y los extranjeros", en M. Albadalejo (Dir.), Comentarios al Código Civil y compilaciones forales, Edersa, Jaén, Tomo 1, p. 749; A. Álvarez Rodríguez, Nociones básicas...op cit, p. 263; J. Peré Raluy, Derecho del Registro Civil, Aguilar, Madrid, 1962, p. 608. En sentido contrario, al considerar que el efecto es meramente declarativo de una situación jurídica que ya existe, Vid J. M. Espinar Vicente/ M. Guzmán Peces, La nacionalidad y la extranjería en el sistema jurídico español, Dykinson, Madrid, 2017, p. 102.

${ }^{12}$ Resulta difícil encontrar apoyo a esta idea en la doctrina, al menos de forma expresa y por escrito, por lo que merece destacar el análisis que en esta misma línea realizan J. M. Espinar Vicente/ M. Guzmán Peces, La nacionalidad y la ...op cit, p. 102.

${ }^{13}$ Vid los comentarios realizados a esta sentencia por C. PÁramo de SANTIAGO, "Nacionalidad española de origen: pérdida y conservación. Comentario a la STS de 19 de diciembre de 2019”, Revista CEF Legal, 230, marzo 2020, pp. 93-98
} 
14. Está claro para el TS que de la lectura del citado artículo no se desprende formalidad específica para formular la declaración de conservación de la nacionalidad española, lo que implica que no está sujeta a una forma solemne y, de acuerdo con la teoría general de las declaraciones de voluntad, en ausencia de norma que imponga determinada solemnidad, no se ve inconveniente para admitir que la voluntad se manifieste de manera indirecta a través de un comportamiento concluyente.

En el caso litigioso, el demandante compareció ante el Consulado para solicitar la renovación de su pasaporte dentro del plazo de 3 años previsto en el artículo $24.3 \mathrm{CC}$ y si bien formalmente no declarara de manera expresa y directa su voluntad de conservar la nacionalidad española, hay que reconocer que su solicitud de renovación de pasaporte debe ser tenida como tal (y no una vez, sino en dos ocasiones) en atención a lo que significa habitual y socialmente en este ámbito la tenencia del pasaporte.

15. En este ámbito, nos recuerda que sólo los españoles tienen derecho a que se les expida un pasaporte y, puesto que se trata del documento que acredita la nacionalidad de los españoles fuera de España (conforme a lo dispuesto en el artículo 11 de la Ley de seguridad ciudadana ${ }^{14}$ ) con carácter "público, personal e intransferible", no puede negarse a su petición el valor jurídico de querer conservar la nacionalidad por parte de quien la formula.

Por ello, la Sala concluye con acierto, que la solicitud de renovación del pasaporte no es un mero uso de la nacionalidad española, sino una petición que comporta de manera inequívoca la voluntad de querer ser español.

16. Con este nuevo pronunciamiento el TS da respaldo a la que ya puede ser considerada como la "nueva doctrina jurisprudencial" que concede a la solicitud de renovación del pasaporte, el valor jurídico correspondiente a la voluntad del español de origen de conservar dicha nacionalidad.

Frente a una visión estrictamente formalista del contenido de la declaración de voluntad conservativa y del concreto órgano consular ante el que presentarla, la Sala opta por una interpretación en la que prima la verdadera intención del afectado de conservar su nacionalidad española mediante un acto, como la solicitud de renovación por segunda vez del pasaporte español, que demuestra inequívocamente dicha voluntad.

\section{Comentario final}

17. En esta sentencia, el TS confirma la interpretación flexible de una norma que puede conllevar a la pérdida de la nacionalidad española de origen a españoles nacidos en el extranjero y es alentador ver que corren nuevos tiempos para la práctica judicial española que, a la larga, influirá en la práctica administrativa desde sede registral y también en la interpretación dada a este precepto desde la Abogacía del Estado español.

18. Reflexionar sobre las causas de pérdida de la nacionalidad española es un ejercicio que permite repensar la conexión entre la nacionalidad de origen y el "blindaje" que la propia Constitución ha pretendido darle desde su entrada en vigor en 1978. Quizás para un estudio doctrinal, y atendiendo a los cambios legales que se avecinan en cuanto a la posible adquisición de la nacionalidad española por parte de los descendientes de españoles de origen, convenga llevar a cabo el análisis que va más allá de los comentarios a vuelapluma que han motivado esta sentencia.

19. Cuando hace unos cuantos años leía a la profesora Álvarez RodRíGUEZ, en una breve nota con respecto a este tema (publicada en el año 1997) pensaba en la capacidad de análisis, previsión y flexibilidad de sus razonamientos en contraposición con la actuación mecánica de los Consulados Españoles y la consecuente actuación de la DGRN en la línea dura de pensamiento y de práctica.

${ }^{14}$ Ley Orgánica 4/2015, de 30 de marzo, de protección de la seguridad ciudadana. BOE num. 77, 31 de marzo de 2015. 
En esa ocasión mis pensamientos volaron hacia el futuro y pensaba que en algún momento las palabras de la profesora se verían reflejadas en una STS y cito: "la mera aceptación y goce de una nacionalidad extranjera no puede por sí misma ser causa de pérdida de la nacionalidad española [de origen], puesto que ello vulneraría la Constitución... por eso, comparecer como español en el consulado y otras conductas semejantes son índice de que el interesado no habrá incurrido en la pérdida de la nacionalidad española.." Pues bien, el futuro ya ha llegado, más de 20 años después. ${ }^{15}$

${ }^{15}$ Extraído de A. Álvarez RodríGuez, "La pérdida de la nacionalidad española (y II)”, Revista de Emigración e Inmigración, num. 513, 1997, p.27. 